\title{
Los desafíos de la salud laboral: problemas históricos y contemporáneos
}

\author{
The challenges of occupational health: \\ historical and contemporary problems
}

\author{
María Fernanda Vásquez \\ Becaria postdoctoral, Programa de Pós-graduação Interdisciplinar em Ciências Humanas/ \\ Universidade Federal de Santa Catarina. \\ Florianópolis - SC - Brasil \\ mfdavasquez@gmail.com
}

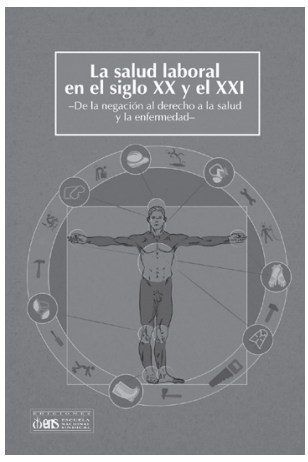

GALLO, Oscar; CASTAÑO, Eugenio (Ed.). La salud laboral en el siglo XXY el XXI: de la negación al derecho a la salud y la enfermedad. Medellín: Escuela Nacional Sindical. 2016. 504p.

$\mathrm{E}$ l libro La salud laboral en el siglo XX y el XXI: de la negación al derecho a la salud y la enfermedad, organizado por los historiadores e investigadores colombianos Oscar Gallo y Eugenio Castaño (2016), es el resultado de algunos de los debates y reflexiones acerca de los derechos en salud, los mecanismos de defensa de derechos y negociación y los riesgos psicosociales en el ámbito laboral, llevados a cabo en 2015 durante el primer Encuentro Internacional de Salud y Trabajo en Colombia, realizado por la Escuela Nacional Sindical.

La iniciativa de elaborar un libro que compilara algunos de los debates históricos y contemporáneos más importantes alrededor de la salud laboral en Colombia y América Latina se hizo extensiva a otros investigadores de diversas partes del mundo interesados en la temática. De ese modo, el libro reúne una completa colección de textos de investigadores de Colombia, Chile, Brasil, Argentina, México, Francia, Alemania, Italia y España, teniendo la historia como denominador común; como herramienta de análisis, la globalización de las problemáticas y la reflexión interdisciplinar.

En los 15 artículos que componen el libro, los investigadores evidencian, de manera cuidadosa y profunda, las múltiples dimensiones de la salud laboral, su entramado legislativo y las luchas sociales y sindicales, a lo largo del siglo XX y XXI en diversos contextos locales, así como la integración de tales problemáticas en el proceso más general de la globalización y de las políticas internacionales.

De esta obra es importante resaltar tres aspectos que hacen ineludible su lectura no solamente para quienes se dedican a la historia del mundo laboral, sino también para aquellos que se interesan en la historia social de la salud y la enfermedad. Esos tres aspectos se refieren, en primer lugar, al surgimiento de nuevos interrogantes y de nuevas formas de abordar el tema de la salud laboral, por lo menos en dos perspectivas: (1) La necesidad de analizar las problemáticas locales vinculadas estrechamente con procesos globales

http://dx.doi.org/10.1590/S0104-59702018000100018 
e internacionales y (2) la urgencia de analizar el problema de la salud y la enfermedad de los trabajadores más allá de la historia de la medicina para integrarlo en un contexto social, político, legislativo, cultural y económico más amplio y complejo. En ese sentido, la descripción de las particularidades locales es utilizada para pensar problemas más generales del mundo laboral y aportar nuevos argumentos a la lucha por la defensa de los derechos de los trabajadores.

Un segundo aspecto que vale la pena resaltar es la orientación teórica y metodológica utilizada por los organizadores del libro. Se trata de un texto en el que el uso de la historia como herramienta de análisis no se restringe a la mera descripción cronológica de acontecimientos o a la colección exhaustiva de datos históricos, sino a un tipo de historia crítica que permite pensar los problemas actuales de la salud laboral a la luz de los acontecimientos históricos que les dieron vida, de analizar los cambios y las permanencias de determinados problemas y sus repercusiones en el mundo laboral. De ese modo, el problema de la enfermedad no se piensa solo desde un punto de vista biológico, sino que se integra en el análisis de problemáticas más generales como la institucionalización de la medicina laboral, la lucha civil por el derecho a la salud y los juegos de poder económicos y políticos para la gestión y organización del trabajo. En consecuencia, la perspectiva interdisciplinar dada al libro refuerza la idea de que la salud y la enfermedad son fenómenos biológicos, sociales y culturales, que deben analizarse de manera global para comprender en profundidad las similitudes y las diferencias de los procesos históricos, de las transformaciones de los actores y de los escenarios de lucha en cada contexto específico.

Y el tercer aspecto se refiere a la cantidad de temáticas abordadas en las tres secciones del libro: "De la negación al derecho a la salud", "El factor humano en la industria" y "Derechos, luchas y realidades". Los estudios de la primera parte muestran cómo el reconocimiento de algunas enfermedades y accidentes de trabajo precisó de un conjunto de cambios legislativos, médicos y en la concepción del cuerpo del trabajador. La segunda parte indaga la dimensión psicológica del trabajador y la manera cómo esta se incorporó en el mundo laboral y en la gestión de los riesgos en el trabajo. La tercera y última parte se concentra en evidenciar las relaciones entre derecho, salud y riesgo en la era del capitalismo flexible a través del análisis de algunos casos como la red Vida Viva de Brasil o Asotrecol de Colombia.

En general, a lo largo de esas tres secciones, los artículos abordan temas relacionados con el cuerpo de los trabajadores, las enfermedades y accidentes de trabajo, la comprensión de la idea de factor humano, los problemas relacionados con la fatiga en el trabajo y su influencia en el desempeño físico y la salud mental, así como temas más actuales sobre los riesgos psicosociales en el trabajo y la rehabilitación profesional. Del mismo modo, se destacan los artículos que hacen énfasis en la relación capital, trabajo y salud, los debates administrativos, legislativos y económicos relativos a la lucha por los derechos, la seguridad laboral y las condiciones de trabajo. Además de aquellos artículos que abordan aspectos como la división sexual del trabajo, el derecho a enfermarse, a la indemnización y las estrategias de resistencia frente al control del espacio laboral.

De manera que, este libro se constituye en un aporte teórico y metodológico relevante para repensar las múltiples facetas de las relaciones entre la salud y el mundo laboral en 
Latinoamérica y Europa, no apenas desde el punto de vista histórico sino también actual, especialmente en una contemporaneidad marcada por el capitalismo flexible y la pérdida de derechos laborales.

Finalmente, considerando el foco del libro, sería interesante, en el caso de publicaciones futuras, indagar sobre la manera en que ciertas instituciones multilaterales económicas internacionales, como el FMI, el Banco Mundial, o locales, como el Mercosur, el Banco Interamericano de Desarrollo (BID), han utilizado diversas estrategias desde los años 1980 para homogenizar determinadas políticas en salud, en respuesta a una pretendida optimización de la relación entre costos y beneficios. Analizar detenidamente como tales cambios han modificado la salud laboral en América Latina constituiría una promisoria línea de estudios sobre la que aún es necesario indagar en profundidad.

\section{REFERENCIA}

GALLO, Oscar; CASTAÑO, Eugenio (Ed.).

La salud laboral en el siglo XX y el XXI: de la negación al derecho a la salud y la enfermedad. Medellín: Escuela Nacional Sindical. 2016. 